\section{Validity of forced expiratory flow volume loops in neonates}

Sir,

Despite the comments of LeSouëf and Landau on our methodology in the Archives, ${ }^{1}$ we remain concerned about the validity of forced expiratory flow volume loops in neonates. ${ }^{2}$ We initially examined the effects of compression pressures ranging from 1.0 to $4.5 \mathrm{kPa}$ and found that pressures above $3 \mathrm{kPa}$ did not lead to significantly higher flows of functional residual capacities in our normal healthy full term babies. We are not competent to comment on what pressures should be used in older infants with airways obstruction but are worried rather than reassured that there is no single pressure which can be used for all infants. It is, presumably, necessary to study each infant over a wide range of compression pressures in order to detect the optimal. Presumably any effective therapeutic manoeuvre could alter the lung mechanical characteristics of the respiratory system to such an extent that this pressure no longer applied, so that the full range of pressures would again have to be used, making this a very difficult and cumbersome technique to use in practice.

We are also concerned that there appear to have been only three previous measurements on the transmission of the pressure to the pleural space. ${ }^{3}$ The authors, Taussig $e t$ $a l$, found that only $25 \%$ of the applied pressure was transferred, a surprisingly low figure when all previous measurements have suggested that the chest wall is far more compliant than the lung in the neonatal period. These figures do, however, fit in well with our own conclusions that the baby is making inspiratory efforts from soon after the onset of the compression pressure and, indeed, analysis of our traces has shown that on all occasions the oesophageal pressure had fallen from its peak value before the functional residual capacity point had been reached in the 188 squeezes. As the transfer pressure is so small, we also think it unlikely that the supramaximal flows for squeezes applied during expiration are caused by flow transcience from compression of the gas in the large airways as claimed by LeSouëf and Landau.

In summary, we are not claiming that this technique is without value but are concerned that the measurements obtained often do not represent flow limitation. This will inevitably considerably reduce sensitivity and may affect the interpretation given on changes (or lack of changes) occurring after therapeutic manoeuvres.

\footnotetext{
References

${ }^{1}$ LeSouëf PN, Landau LI. Validity of forced expiratory flow volume loops in neonates. Arch Dis Child 1988;63:460-1.

${ }^{2}$ Hoskyns EW, Milner AD, Hopkin IE. Validity of forced expiratory flow volume loops in neonates. Arch Dis Child 1987;62:895-900.

3 Taussig LM, Landau LI, Godfrey S, Arad I. Determinants of forced expiratory flow in newborn infants. J Appl Physiol 1982;15:1270-7.

E W Hoskyns, A D Milner, and I E Hopkin Department of Child Health, University Hospital, Queens Medical Centre, Nottingham NG7 $2 U H$
}

\section{Comparison of five tests used in diagnosis of neonatal bacteraemia}

Sir,

We have read with interest the paper by Kite et al. ${ }^{1}$ The authors have obtained the best sensitivity $(61 \cdot 8 \%)$, specificity $(90 \cdot 3 \%)$, and positive predictive accuracy $(56 \cdot 8 \%)$ using a combination of the acridine orange leucocyte cytospin test, C reactive protein assay, and nitroblue tetrazolium test. Moreover, they have showed improvement of the sensitivity $(92 \cdot 3 \%)$ excluding bacteraemia with coagulase negative staphylococci (21/34 episodes of bacteraemia). Unfortunately, the authors have not pointed out the negative predictive accuracy of the evaluated teststhat is, the probability that sepsis is not present if the tests are negative.

Recently, we have evaluated the usefulness of seven tests (neutrophil blood cell count, the ratio of band forms to total neutrophils, the platelet count, and the quantitative determination of serum IgM, C reactive protein, alpha-I-acidglycoprotein, and haptoglobin) for the early identification of the serious neonatal infections. ${ }^{2}$ We have prospectively studied 70 preterm newborns: 15 with sepsis, two with serious infections without bacteraemia, and 53 without infections. When two or more tests were positive, sensitivity was $76.4 \%$, specificity was $83 \%$, positive predictive accuracy was $59 \%$, and the negative predictive accuracy was $91 \cdot 6 \%$.

Previously, Philip and Hewitt have reported (using band/total neutrophils, leucocyte count, latex $\mathrm{C}$ reactive protein, erythrocyte sedimentation rate, and latex haptoglobin) that, when two or more tests were positive, the positive predictive accuracy was $39 \%$ (62\% considering 'very probable' sepsis); on the other hand, when less than two tests were positive, the probability that sepsis was not present was $99 \%$ (negative predictive accuracy). ${ }^{3}$

In our opinion, values of positive predictive accuracy as $56.8 \%,{ }^{1} 59 \%,{ }^{2}$ or $39-62 \%{ }^{3}$ are unacceptable in predicting a severe disease as neonatal sepsis. On the contrary, we want to underline the great potential usefulness of the negative predictive accuracy of the evaluated tests $^{2}{ }^{3}$ to exclude infection and avoid antibiotics when the diagnosis of sepsis on clinical grounds is doubtful.

\section{References}

${ }^{1}$ Kite P, Millar MR, Gorham P, Congdon P. Comparison of five tests used in diagnosis of neonatal bacteraemia. Arch Dis Child 1988;63:639-43.

2 Stabile A, Pastore M, Romanelli V, Miceli Sopo S, Flamini G, Pesaresi A. Diagnosi precoce di sepsi nel neonato pretermine. Pediatr Med Chir 1987;9:3-4.

${ }^{3}$ Philip AGS, Hewitt JR. Early diagnosis of neonatal sepsis. Pediatrics 1980;65:1036-41.

S Miceli Sopo, M A Pesaresi, M Pastore, and A Stabile Clinica Pediatrica dell'Università Cattolica del Sacro Cuore, Largo Gemelli 8, 00168, Roma, Italy 